Original Article

Gopal R Sharma, MS

Department Of Neurosurgery

National Academy of Medical Sciences (NAMS)

Bir Hospital, Kathmandu, Nepal

Prakash Bista, MS, MCh

Department Of Neurosurgery

National Academy of Medical Sciences (NAMS)

Bir Hospital, Kathmandu, Nepal

Nilam Khadka, MCh

Department Of Neurosurgery

National Academy of Medical Sciences (NAMS)

Bir Hospital, Kathmandu, Nepal

Rajiv Jha, MS, MCh

Department Of Neurosurgery

National Academy of Medical Sciences (NAMS)

Bir Hospital, Kathmandu, Nepal

Maya Bhattachan, MD, MCh

Department Of Neurosurgery

Nepal Medical College

Jorpati, Kathmandu, Nepal

Sumit Joshi, MS

Department Of Neurosurgery

Nepal Medical College

Jorpati, Kathmandu, Nepal

Pawan Kumar, MS

Department Of Neurosurgery

Nepal Medical College

Jorpati, Kathmandu, Nepal

Corresponding address:

Gopal Raman Sharma, MS

Department of Neurosurgery

National Academy Of Medical Sciences (NAMS)

Bir Hospital, Kathmandu, Nepal

Email: gopa147@hotmail.com

Received, 24 March, 2015

Accepted, 9 April, 2015
Nepal Journal of Neuroscience 12:31-37, 2015

\section{Our Experience with Infratentorial Supracerebellar (ITSC) Approach for Pineal Region Tumor Excision}

Pineal region tumors are rare and their incidence is less than $1 \%$ of primary CNS tumors. Varieties of tumors can be found in pineal region ranging from benign to malignant pathology. Treatment modalities ranged from biopsy to total excision of tumor. Many surgical approaches have been described in the literature, however, infratentorial supracerebellar ( ITSC ) approach is versatile and widely used by many neurosurgeons worldwide.

The aim of this study is to analyze and discuss the surgical outcome of pineal region tumor using ITSC approach. Advantages and limitations of this approach will be discussed.

This is a retrospective study of 10 patients who underwent microsurgical excision of pineal region masses using ITSC approach in sitting position under general anesthesia at our institute between April 2009 and March 2014. The follow up period ranged from 7 months to 5 years and outcome was measured with GOS (Glasgow Outcome Scale).

There were 9 male and 1 female and age ranged from 10 to 50 years.

Gross total resection was performed in all 10 cases using ITSC approach. 9 patients required VP shunt for obstructive hydrocephalus. 60\% (6) had benign and 40\% (4) had malignant pathology.

Six patients suffered from postoperative complications which included air embolism ,pneumocephalus, pseudomeningocele and seizure.

Eighty percent (8) have good recovery, 1 was severely disabled and 1 died.

Advanced neurological techniques combined with neuroanesthesia, neuroimaging and postoperative critical care methods have made aggressive surgical resection a mainstay of management of pineal region tumors with excellent results.

Key Words: infratentorial supracerebellar (ITSC) approach, pineal region mass, sitting position, surgical excision
$\mathrm{P}$ ineal region tumors are rare, accounting for less than $1 \%$ of all primary CNS tumors, with somewhat higher percentages in Japanese population. Pineal tumors are common in children and young adult. ${ }^{2,13}$
Tumors of pineal region represent a diverse collection of tumors which may involve not only pineal gland itself but also posterior portion of the third ventricle, tectum and aqueduct of mid brain. ${ }^{13}$ There are four groups of tumors 


\section{Sharma et al}

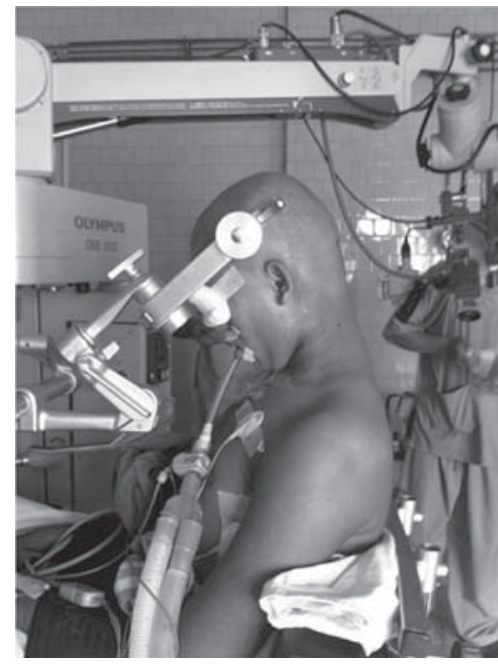

Figure 1: Patient in sitting position for ITSC approach

in pineal region and they are germ cell tumors, pineal parenclymal tumors, glial tumors and tumors of other histology like meningioma, epidermoid, etc. ${ }^{12,30}$

Given the diverse pathology that can occur in the pineal region, a histologic diagnosis is necessary to optimize management decisions. Treatment modalities include tumor biopsy to total excision of tumor . 1,2,4,9,11,14,26,28,31

There are several surgical approaches for open surgery to pineal regions tumor, however, infratentorial supracerebellar (ITSC) approach has been widely accepted and used worldwide and about $90 \%$ of pineal region tumors can be removed using this approach. 3,16,20,22,23,32

Oppenheim and Krause reported the first successful pineal region tumor removal in 1913 using ITSC approach. 21 In 1926, Krause described modest success without operative morbidity in three patients using ITSC corridor. Then this surgical approach was lost for five decades due to limitations of microsurgical techniques. In 1971, Stein 32 reintroduced and popularized this approach for excision of pineal region tumors using operating microscope and microinstruments with operative mortality of only $3 \%$. $^{2,3}$

Two third of pineal region tumors are malignant and they give good response to radio and chemotherapy. Especially, germ cell tumors are very sensitive to radiotherapy and 10 year survival is about $95 \%$ after radiation. ${ }^{17,18}$

\section{Results}

This is a retrospective study of 10 cases who were operated for pineal region tumors using ITSC approach in sitting position (Figure 1) over a period of five years, between April 2009 and March 2014, in our institute ( Table 1).

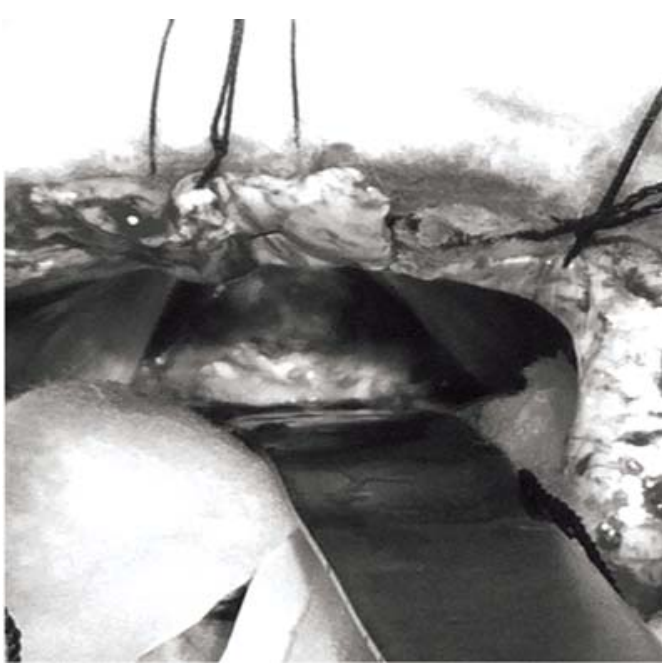

Figure 2: Peroperative picture showing the pineal region pearly white mass (epidermoid) via ITSC corridor.

There were 9 male and 1 female. Age ranged from 10 to 50 years.

Follow up period ranged from 7 months to 5 years.

On admission, all patient had features of raised intracranial pressure i.e. headache, vomiting and papilloedema on funduscopy, due to obstructive hydrocephalus. 4 patients had impaired vision. 3 patients had parinaud signs and 3 had cerebellar signs.

All tumors were approached via ITCS corridor in sitting position (Figure 2), however, in one case, patient needed second procedure who had tumor extension to superior region, above the tent, and required occipital transtentorial approach to take out remaining part of meningioma.Diagnosis were made either by cranial CT scan and or MRI with intravenous contrast (Figures: $\mathbf{3 a}, \mathbf{3 b}$ and $\mathbf{4 a}, \mathbf{4 b}, \mathbf{4 c}$ ). Tumor markers were negative in serum and CSF in all cases. Gross total excision of tumors was performed in all cases.

Nine patients required ventriculoperitoneal shunt for obtructive hydrocephalus prior to major surgical undertaking.

Histologically, 3 patients had ependymoma, 3 had meningioma, 2 epidermoid ,1 pineoblastoma and 1 pilocytic astrocytoma.

Four patients required postoperative cranial radiation who had malignant pathologies.

Six patients suffered postoperative complications; 2 had seizures, 2 had pneumocephalus, one had intraoperative air embolism and one had pseudomeningocele.

Good recover was achieved in $80 \%$ (8), one was severely disabled (10\%) and one died (10\%).

On postoperative MRI of brain with contrast there was no tumor recurrence in our series till to date (Figure 3c, 3d and 4d,4e). 


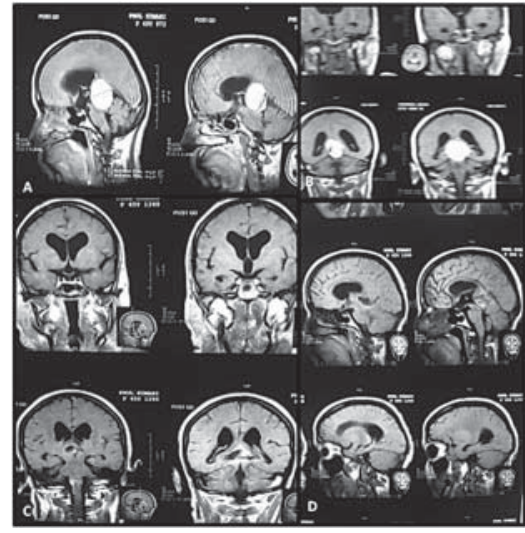

Figure 3: A and B, Preoperative MRI of brain with Gadolinium enhancement depicting enhancing solid pineal region mass suggestive of meningioma. $C$ and $D$, Postoperative MRI pictures with IV contrast showing no evidence of residual tumor

\section{Discussion}

Tumors of the pineal region represent a diverse collection of tumors which may involve not only pineal gland itself but also posterior portion of the third ventricle, tectum and aqueduct of mid brain.

Pineal region tumors are rare which accounts only $1 \%$ of all primary central nervous system(CNS) tumors, with significantly higher rate in Japanese population. Pineal region tumors are the tumors of children and young adults. ${ }^{2,13}$

WHO has classified pineal region tumors into four groups and they are,

a) germ cell tumors, b) pineal parenchymal tumors c), glial tumors and d) tumor of other histology. $8,12,30$

Germ cell tumors account about $30 \%$ of all pineal region tumors which includes germinoma, embryonal cell carcinoma, yolk sac tumor, choriocarcinoma and teratoma.

Pineal parenchymal tumor represents about $30 \%$ of primary pineal region tumors and they are pineocytoma and pineoblastoma.

Glial tumors represents another $30 \%$ of pineal tumor and they are ependymoma, pilocytic astrocytoma, etc.

Other $10 \%$ of pineal region tumors are miscellaneous and they are meningioma, dermoid, epidermoid, arachanoid cyst, etc.

Two third of pineal region tumors are malignant and one third are benign. ${ }^{12,28}$

Majority of patients harboring pineal region tumors present to clinicians with the features of raised ICP due to obstructive hydrocephalus. ${ }^{1,2,4,26}$ Some of them may present with parinaud syndrome due to the compression of tectal plate of midbrain. Parinaud syndrome is a combination of upward gaze palsy, convergence or refraction nystagmus

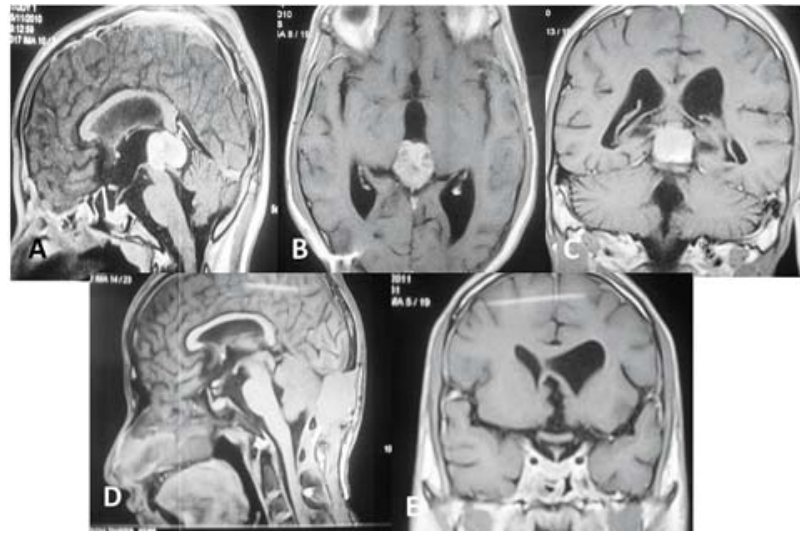

Figure 4: A, B and C, Preoperative MRI of head of 15 year old boy showing the brightly enhancing pineal region mass. D and E, Postoperative MRI of brain showed no residual tumor. Histology was ependymoma

and light near pupillary dissociation. Patients may also develop cerebellar or brain stem compression signs. Few may have features of endocrine dysfunction like diabetes insipidus and precocious puberty. ${ }^{13,31}$

Our patients, in this series, also had similar clinical presentations as described in literature, however, there was no features of endocrine dysfunction.

Best diagnostic tool to diagnose pineal region tumor is MRI of brain with gadolinium contrast, however, these days, due to availability of multi slice, multi planner CT Scan with contrast, CT scan is equally helpful to come to diagnosis and plan surgery.

Elevation of tumor markers in serum and CSF might be helpful to come to the diagnosis of some of the pineal region tumor. ${ }^{2,8,13,31} \mathrm{~B}-\mathrm{HCG}$ in serum and CSF is elevated in germinoma and choriocarcinoma, likewise, Alfa-fetoprotein is elevated in endodermal sinus tumor. Melatonin and S-antigen might be raised in pineoblastoma. Raised tumor marker level in CSF is more specific than in serum, because markers in serum might be elevated in tumors of the other part of the body.

We had measured the tumor marker level in serum and CSF in all our patients but did not find any elevation of specific tumor marker.

Given the diversity of pathology that can occur in the pineal region, a histologic diagnosis is necessary to optimize management decision. Surgical treatment of pineal region tumors is thought to be one of the most challenging neurosurgical problem. In fact, pineal body lies within the geometrical centre of the brain surrounded by critical neurovascular structures which damage may be fatal. So, pineal region surgery is among the most arduous of microsurgical challenges and outcomes vary substantially with the expertise of individual surgeon. 


\section{Sharma et al}

In 1932, Harvey Cushing wrote that he "never succeeded in exposing a pineal tumor sufficiently well to justify on an attempt to remove it". ${ }^{13}$

Management of pineal region tumors consisted of treatment of hydrocephalus and tissue diagnosis. More than $90 \%$ of patients who have pineal region tumors develop hydrocephalus due to obstruction and require CSF diversion surgery either before or after major surgical undertaking.,2,8,13 Tissue diagnosis can be made either stereotactic or endoscopic biopsy or open surgical proce dure. ${ }^{1,2,4,8,13,15,17,19,20,22,23,26,28,31,32}$

These days, due to advancement in neurosurgical techniques combined with neuroanesthesia and postoperative critical care methods have made aggressive surgical resection, a mainstay of management. So, complete tumor removal has been clearly established an optional treatment for pineal tumors. $2,7,11,14,16,20$

Surgical resection can provide; 1) tissue diagnosis with a greater diagnostic yield than stereotactic biopsy, 2) total cure with benign lesions, 3 ) cytoreduction to improve the efficacy of chemotherapy and radiotherapy and 4) restoration of the CSF flow through the removal of the tumors block.

There are several surgical approaches to reach and excise the pineal region tumors, but the best approach to use, depends on the anatomic location, spread of the tumor(extension), a degree of familiarity and confidence to the surgeon and size of the tumor.

Commonly used approaches for pineal region tumors are infratentorial supracerebellar (ITSC), occipital transtentorial, transcallosal interhemispheric and transcortical transventricular 2,3,4,8,11,13,16,20,22,23,31,32

The first successful removal of a pineal tumor was reported by Krause and Oppenheim in 1913 and they had removed the tumor using ITSC approach. ${ }^{21}$ Then in 1926, Krause described modest success without operative mortality in three patients. After that, this ITSC technique was lost for many years because of limitation of microsurgical techniques.

In 1921, Walter Dandy described the transcallosal approach he used in three pineal tumor patients. ${ }^{5,6}$

\begin{tabular}{|c|c|c|c|c|c|}
\hline Name & Age/ Sex & Clinical presentation & Approaches & Extent of excision & Histology \\
\hline 1.Prakash Thapa & $15 / \mathrm{M}$ & $\begin{array}{l}\text { Featuresof raised ICP .par- } \\
\text { inaud signs }\end{array}$ & ITSC & Total & Ependymoma \\
\hline 2.Manish Chaudhary & $25 / \mathrm{M}$ & $\begin{array}{l}. \text { Features of raised ICP } \\
. \downarrow \text { Vision } \\
\text {.Gait ataxia }\end{array}$ & ITSC & Total & Epidermoid \\
\hline 3. Bhim Bahadhur BK & 19/M & $\begin{array}{l}. \text { Raised ICP } \\
. \downarrow \text { Vision } \\
. \text { Parinard signs }\end{array}$ & ITSC & Total & Epidermoid \\
\hline 4.Indrajeet Chaudhary & $8 / \mathrm{M}$ & $\begin{array}{l}\text {.Coma } \\
\text {.Raised ICP }\end{array}$ & ITSC & Gross total & Ependymoma \\
\hline 5.Radha Kumar Khadka & $33 / \mathrm{M}$ & $\begin{array}{l}\text {.Parinaud Signs } \\
\text {.Raised ICP }\end{array}$ & ITSC & Gross total & Meningioma \\
\hline 6.Umakant Chaudhary & 7/M & Raised ICP & ITSC & Gross total & Ependymoma \\
\hline 7.Durga Kumari Magar & $20 / \mathrm{M}$ & $\begin{array}{l}\text { Raised ICP } \\
\text {.BIL Blindness }\end{array}$ & ITSC & Gross total & $\begin{array}{l}\text { Pilocytic as- } \\
\text { trocytoma }\end{array}$ \\
\hline 8.Phul Kumari Devi & $43 / \mathrm{F}$ & Raised ICP & ITSC & Total & Meningioma \\
\hline 9.Kale Tamang & $45 / \mathrm{M}$ & Raised ICP & ITSC & Total & Pineoblastoma \\
\hline 10.Dharmendra & $29 / \mathrm{M}$ & $\begin{array}{l}\text { Raised ICP } \\
\text {.BIL diminution of Vision }\end{array}$ & ITSC,OT & Gross total & Menigioma \\
\hline
\end{tabular}

$\mathrm{ICP}=$ Inracranial pressure; ITSC = Infratentorial Supracerebellar; OT= Occipital Transtentorial

Table 1: Summary of all patients who underwent open surgical resection for pineal region tumors via ITSC approach in sitting position 
Pineal Region Tumor

\begin{tabular}{|l|l|l|l|}
\hline Authors & Year & No. of Cases & Mortality ( \% ) \\
\hline Dandy & 1936 & 10 & 70 \\
\hline Horrax & 1950 & 10 & 50 \\
\hline Rand and et al & 1953 & 17 & 70 \\
\hline Ringutz & 1954 & 51 & 59 \\
\hline Icuniclci & 1960 & 8 & 25 \\
\hline Poppen and Marino & 1968 & 9 & 44 \\
\hline
\end{tabular}

Table 2: Mortality of pineal region mass surgery before microsurgical era.

Masses after the use of microsurgical technique.

\begin{tabular}{|l|l|l|l|l|}
\hline Authors & Years & No. Of Patients & Morbidity (\%) & Mortality (\%) \\
\hline Stein & 1971 & 6 & 0 & 0 \\
\hline Page & 1977 & 9 & 0 & 11 \\
\hline Jooma and Kendall & 1983 & 20 & 0 & 0 \\
\hline Kobayashi et al & 1983 & 14 & 0 & 0 \\
\hline Sano & 1985 & 32 & 0 & 0 \\
\hline Bruce and Stein & 1992 & 141 & 3 & 4 \\
\hline
\end{tabular}

Table 3 : Results following infratentorial supracerebellar excision of pineal region

The occipital transtentorial approach was first described by Horrax in 1937 and later modified by Poppen in 1960 and by Jamaieson in $1971.9^{9,10,24}$

Mortality from pineal region tumor surgery before microsurgical era was very high which ranged from $44 \%$ to $70 \%$ (Table 2), however, after 1970 s, the mortality sharply reduced down to $3 \%$ to $10 \%$ (Table 3 ) due to use of operating microscope, microsurgical instruments and techniques and advancement in neuroanesthesia, imaginq and postoperative cure.

In 1971, Stein reintroduced and popularized ITSC approach for pineal tumor excision. ${ }^{32} \mathrm{He}$ used operating microscope and microinstruments while removing tumors and result was excellent and mortality was about $3 \%$ only. ITSC and occipital transtentorial approaches are currently most widely and frequently used access to excise the lesions of pineal region.

This ITSC corridor is widely accepted and used by many neurosurgeons around the world which is safe and effective and permits total excision of the tumor of the pineal region in the most of the cases. . $3,7,16,20,22,23,32^{2}$

ITSC is indicated for small and medium sized pineal region tumor located infratentorially and in midline. This approach is performed either in sitting or in prone (Concord) position. Majority of surgeons prefer to do it in sitting position, because gravity works in surgeon's favor by reducing the pooling of blood and CSF in the operative field and by facilitating the dissection of the tumor from the deep venous system. The advantages of
ITSC approach are minimal damage to neural tissues, and allowing the surgeon to avoid crossing the deep venous system. The disadvantages of ITSC approach are narrow operating space, difficult to remove the part of the tumor which has extended laterally, superiorly and inferiorly. Complications related with sitting positions are intraoperative air embolism, pneumocephalus and subdural hematoma.

Air embolism is a serious complication and incidence rate is about 7 to $25 \%$. It might be fatal if it is not diagnosed and treated in time. ${ }^{4,8,13}$

In our study, one patient had developed air embolism during surgery which was promptly diagnosed and treated by our experienced neuroanesthetists.

Optimal treatment of pineal region tumors, as mentioned earlier, should be radical excision, when possible, to have total cure if benign lesion and to improve the response to chemo and radiotherapy if they are malignant tumors. Additionally total excision of tumor might reopen the CSF pathways and resolve hydrocephalus and CSF diversion surgery can be avoided.

In Oliveira series of 32 patients who were operated for pineal region tumor via ITSC approach total removal was achieved in 15 cases, subtotal in 7 patients and only partial removal was possible in 10 patients. ${ }^{22}$ There was total resection of tumors in 14 patients $(82.3 \%)$, subtotals in 2 patients(11.8\%) and only one biopsy in $1(5.9 \%)$ in Maichzzak's series of 17 patients who harbored pineal tumors. ${ }^{16}$ 


\section{Sharma et al}

In Konovalov's Series of 225 patients who underwent surgical excision, total tumor removal was achieved in $148(58 \%)$, subtotal in $74(29 \%)$ and partial in 33(13\%). ${ }^{14}$

All 10 patients, in our series, had gross total excision of tumors via ITSC approach, in sitting positions.

About $90 \%$ of patients with pineal region mass present with associated obstructive type of hydrocephalus which may require CSF diversion surgery either before or after surgical excision of tumors. In our series, 9 patients $(90 \%)$ required ventriculoperitoneal ( VP ) shunt prior to major surgical undertaking, who presented with features of raised ICP and compromised GCS (Glasgow Coma Score).

There were 6 patients $(60 \%)$ with benign and 4 patients ( $40 \%)$ with malignant lesions in our series. The patient with benign tumors included 3 meningiomas, 2 epidermoids and 1 pilocyctic astrocytoma. The patient harbouring malignant lesions were 3 ependymomas and 1 pineoblastomas . Our histological findings are just opposite to previous experiences which had shown malignant tumors in two third of the cases and benign tumors in one third of the cases. ${ }^{4,8,13,28}$ These differences might be due to small number of cases in our series. We have to wait and add more cases to come to final conclusion. ${ }^{4}$ patients who had malignant pathology were subjected for a course of cranial radiation. Overall postoperative complications rate in our series is $60 \%$ (6) which is significantly higher than the complication rate of previously published series of pineal region tumor surgery.

One patient suffered intraoperative air embolism which was managed effectively by anesthetists. CT Scan of brain of 2 patients showed pneumocephalus postoperatively which resolved spontaneously and did not require any further surgical intervention. 2 patients developed seizure after surgical treatment which might be due to VP Shunt insertion but not related to tumor excision. They were treated with anticonvulsants.

Pseudomeningocele was developed in 1 patient which was treated with regular lumbar drain for 1 week.

In Bruce and Stein series of 107 cases, morbidity was observed in 3\%.3 In Hancq series of 8 patients, one patient had developed parinaud syndrome. ${ }^{7}$ There was $13.4 \%$ (2) morbidity in Maichvazok's series of 15 patients. ${ }^{16}$

There was no morbidity and mortality in Konovalvov's and Pluchino's series of 87 and 16 patients respectively. 14,25

In Oliviera series of 32 patients, 56\% (18) suffered post operative complications. ${ }^{22}$

Post operative complications rate in this series is higher than the complications rate of previously published series, this might be probably due to small number of cases and learning curve of operating neurosurgeon. True result might come in coming years when more number of cases would be added.

There were $0 \%$ mortality in Oliveiva, Mottolese, Maichvzale,Pluchino and Konovalvov's series. ${ }^{14,16,20,22,25}$

$4 \%$ mortality was observed in Bruce's Series of 107 cases. 3 Likewise, $12.5 \%$ (1) died due to surgery related complications in Hancq's series of 8 patients. ${ }^{7}$

Our study showed $10 \%$ mortality, which is comparable with previous published series of pineal region tumors.

As it is true that recurrence of tumor depends upon extent of tumor excision and pathology of tumor.

So far, in our series, we have not observed any evidence of recurrence of tumor on follow up imaging of brain of our patients till to date (Figure 3c,3d and 4d,4e).

\section{Conclusions}

Advanced microsurgical techniques combined with neuroimaging, neuroanesthesia and postoperative critical care have made aggressive surgical resection, a mainstay of management of pineal tumors with excellent results.

\section{References}

1. Brockmeyer D, Abtin K, Carey L, Walker M. Endoscopic third ventriculostomy: an outcome analysis. Pediatr Neurosurg 28: 236 -240, 1998

2. Bruce JN, Stein BM. supracerebellar approaches in the pineal regions. In: Brain Surgery : Complication, avoidance and management. Ed. Apuzzo MJL, New York: Churchil Livingstone, pp 511-536,1993

3. Bruce JN, Stein BM. Surgical management of pineal region tumors. Act Neurochir 134: 130-135, 1995

4. Chernov MF, Kamikawa S, Yamane F, Ishihara S, Kubo O, Hori T. Neurofiberscopic biopsy of tumors of the pineal region and posterior third ventricle : Indication, technique, complications and results. Neurosurgery 59: 267-277, 2006

5. Dandy WE. An operation for the removal of pineal tumors. Surg Gynaecol Obstetr 33: 113-119,1921

6. Dandy WE. Operative experience in cases of pineal tumor. Arch Surg 33: 19, 1936

7. Hancq S, De Witte O, Brochi J. Pineal region surgery. Experience in 22 patients. Neurochirgie 48: 14-24, 2006

8. Herrick MK. Pathology of pineal tumors. In : Neuwelf EA (ed): Diagnosis and treatment of pineal 
region tumors, Baltimore: William and Wilkins, pp 31-59, 1984

9. Horrax G. Treatment of tumors of the pineal body. Arch Neurol psychiatry 69: 227, 1950

10. Jamieson KG. Excision of pineal tumors. J Neurosurg 48: 99-102, 1978

11. Jooma R, Kendall BE. Diagnosis and management of pineal tumors. J Neurosurg 58: 654, 1983

12. Kleihues P, Cavenee WK (eds), Tumors of nervous system; pathology and genetics: WHO Classification of Tumors, Lyon, LARC press, 2000

13. Konovalov AN, Pitskhelauri DI. Pineal region tumors. In: essential practice of Neurosurgery. ( Eds) Kalangu KKN, Kato Y, Dechambenoit G .Access publishing Co. Ltd: Nagoya, Japan, pp 371-391, 2011

14. Konovalov AN, Pitskhelauri DI. Principles of treatment of the pineal region tumors. Surg Neurol 59: 250-268, 2003

15. Kreth FW, Shatz C, pagenstecher A, Faist M, Volk B, Ostertag CB. Stereotactic management of lessons of the pineal region, Neurosurgery 39: 280-229, 1996

16. Maichvzak H, Kopera M, Majchvzak K, Kaspera W. surgical treatment of pineal and tectal tumors from the subtentorial and supracerebellar approach. Neurol Neurochir Pol. 34: 899-908, 2000

17. Maity A, Shu HKG, Janss A. Craniospinal irradiation in the treatment of biopsy proven intracranial germinomas : twenty five years experience in a single centre. Int J Radait Oncol Biol Phys 58: 1165-1170, 2004

18. Matsuani M. Sano K, Takakura K, Fujimaki T, Nakamura O, Funata N, Seto T. Primary Intracranial germ cell tumors: a clinical analysis of 153 histologically verified cases. Neurosurgery 86: 446455, 1997

19. Matsutani M. Clinical management of primary central nervous system germ cells tumors. Semin Oncol 31: 676-683, 2004

20. Mottolese C, Szathmari A, Ricci- Franchi AC, Gallo P, Beuriat PA,Capone G. Supracerebellar infratentorial approach for pineal region tumors: Our surgical and technical considerations. Neurochirgie 61: 176-183, 2015

21. Oppenheim H, Krause F. Operative Erfolge bei Geschwulsten der sehhugel- und vierhugelgegend.

Bel klin Wochenschr 50: 2116-2322, 1913

22. Oliveira J, Cerejo A, Silva PS, Polonia P, Pereiva J and Var R. The infratentorial supracerebellar approach in surgery of lessons the pineal region. Surg Neurol Int 4: 154,2013

23. Page LK. Infratentorial supracerebellar exposure of tumors in the pineal area. Neurosurgery 1: 36, 1977

24. Poppen JL, Marino R. Pinealomas and tumors of the posterior portion of the third ventricle. J Neurosurg 28: 357,1968

25. Pluchino F, Broggi G, Fornari M, Franzini A, Solero CL and Allegranza A. Acta Neurochir 96: 26-31, 1989

26. Pople IK, Athanasiou TC, Sandeman DR, Coakham HB. The role of endoscopic biopsy and third ventriculostomy in the management of pineal region tumors. Br J Neurosurgery 15: 305-311, 2001

27. Rand RW, Lemmen LJ. Tumors of the posterior portion of the third ventricle. J Neurosurg 10: 1-18, 1953

28. Regis J, Bouilott P,Rouby-Volot F, FigarellaBranger D, Dufour H, Peragut JC. Pineal region tumors and the role of stereotactic biopsy: review of the mortality, morbidity and diagnostic rates in 370 cases. Neurosurgery 39: 907-914, 1996

29. Ringertz N, Nordenstam H, Flyger G. Tumor of the pineal region. Exp Neurol 13:540, 1954

30. Russel DS, Rubinstein LJ. Pathology of tumors of the nervous system. ed4. Baltimore: Williams and Wilkins pp 284-295,1977

31. Sano K. pineal region and posterior third ventricular tumors : a surgical overview. In: Apu220 MCJ (ed) : Surgery of the third ventricle, Williams and Wilkins, Baltimore ,pp 663, 1989

32. Stein MB. The infratentorial supracerebellar approach to the pineal lesions . J Neurosurg 35: 197, 1971 